\title{
Soledad, rumiación y estrés como precursores del bienestar subjetivo en las parejas de pacientes con cardiopatía
}

\author{
Loneliness, rumination and stress as precursors \\ of subjective well-being in couples of patients with heart disease
}

\author{
Yanitza Nicole Flórez-Rodríguez ${ }^{1}$ y Rozzana Sánchez-Aragón ${ }^{2}$
}

Universidad de Nariño ${ }^{1}$

Universidad Nacional Autónoma de México²

Autor para correspondencia: Yanitza Nicole Flórez R.,nicole.florez.r@gmail.com.

\begin{abstract}
Los objetivos de esta investigación fueron los de identificar las diferencias entre hombres y mujeres, parejas de pacientes con cardiopatía, respecto a la soledad, la rumiación, el estrés y el bienestar subjetivo; la relación entre la soledad con la rumiación y el estrés y, por último, el nivel de predicción de la soledad, la rumiación y el estrés sobre el bienestar subjetivo en una muestra de 130 participantes (65 mujeres y 65 hombres) que tenían una relación de pareja con pacientes con cardiopatía de la Ciudad de México. Los resultados muestran que sentirse dentro de una relación romántica, amistosa y familiar trae consigo menos rumiación y descontrol ante el estrés, pero más satisfacción y prosperidad ante la vida, por lo que, al parecer, la soledad propicia sentimientos negativos - como la rumiación y el estrés- debido a que se hace una evaluación desfavorable sobre el entorno, que se ve aumentado por la excesiva carga física y emocional que deben soportar, pese a lo cual las relaciones cercanas funcionan como fuente de bienestar. De igual manera, se evidencia cómo es que la soledad generada por la enfermedad afecta la evaluación positiva hacia la vida y el control ante las situaciones de estrés, y a la vez protege y potencializa las emociones positivas en los sujetos. Por ello, es de gran relevancia poner atención sobre los cuidadores, ya que su bienestar y calidad de vida se ve comprometida al momento de brindar apoyo al ser querido.
\end{abstract}

Palabras clave: Cardiopatía; Soledad; Rumiación; Estrés; Bienestar subjetivo.

\footnotetext{
${ }^{1}$ Calle Chichimecas 373, Col. Ajusco, Coyoacán, 04300 Ciudad de México, México, tel. (55)12-23-87-20, correo: nicole.florez.r@gmail.com.

${ }^{2}$ Chimalcoyolt 14, Col. Toriello Guerra, Tlalpan, 14050 Ciudad de México, México, tel. (55)41-89-45-44, correo: rozzara@unam.mx.
} 
perceived prosperity in life, and better stress control. These results suggest that loneliness fosters negative feelings associated with a negative evaluation of the surrounding environment and increased excessive physical and emotional burden. However, close relationships function as a source of wellbeing. Results also suggest that disease-related loneliness interacts with perceived quality of life and sense of control in stressful situations and promotes positive emotions. Therefore, care for $\mathrm{ca}$ regivers is of great relevance since wellbeing and quality of life are compromised when providing support to a sick partner.

Key words: Cardiopathy; Loneliness; Rumination; Stress; Subjective wellbeing.

Recibido: $29 / 05 / 2020$

Aceptado: 07/10/2020

L as cardiopatías son la principal causa de defunciones en el mundo (Organización Mundial de la Salud [OMS], 2017) y se definen como un tipo de padecimiento crónico no trasmisible que afecta al corazón y los vasos sanguíneos; asimismo, han mostrado una mayor prevalencia desde mediados del siglo XX, pues de cada mil nacidos, ocho llegan a padecerlas (Valentín, 2018). En el caso de México, el Instituto Nacional de Estadística, Geografía e Informática [INEGI] (2017) señala a estas enfermedades como la principal causa de muertes en el país, constituyendo el $15.7 \%$ de toda la mortalidad.

La presencia de otros padecimientos como hipertensión, hipercolesterolemia, ácido úrico alto, diabetes, obesidad y conductas insanas, como mala alimentación, consumo de tabaco y vida sedentaria (Zavala, Verdejo y Díaz, 2015) aumentan el riesgo de sufrir algún tipo de cardiopatía, lo que ocasiona así mayores complicaciones a la salud de la persona (Jackson, Leslie y Hondorp, 2018).

Pese a todo lo mencionado, el cuidado y sostén que brinden las relaciones interpersonales cercanas hace posible mitigar las reacciones negativas de la enfermedad y superar las adversidades. Es aquí donde la pareja cobra relevancia, al ser uno de los vínculos más importantes para el ser humano y una fuente de soporte y apoyo (Martínez, Fuertes, Orgaz, Vicario y González, 2014), además de que contribuye a suplir las necesidades de ser amado, apreciado y protegido (Sánchez, 2016). Sin embargo, el cuidado de una persona enferma entraña una situación compleja, ya que se debe afrontar y satisfacer las necesidades del miembro dependiente, lo que implica perder o dejar atrás el estilo de vida anterior (Feldberg et al., 2011). En efecto, la pareja de la persona enferma debe hacerse a la idea de que sus responsabilidades se acrecentarán al hacerse cargo de las tareas que implican atender al otro, como su posible incapacidad, el dolor o sus molestias cotidianas, así como llevar a cabo los tratamientos y demás actividades. A partir de esto, surgen sentimientos perturbadores en la pareja del paciente con cardiopatía, como fracasar en la ejecución de dichas tareas, la incertidumbre ante el futuro, la culpa (pues en ocasiones los cuidadores se comprometen tanto que no cumplir debidamente con su deber les genera tal sentimiento) y la soledad al no poder compartir con la pareja una vida "normal", lo que pone en peligro el bienestar de la pareja y la calidad de su relación (Feldberg et al., 2011; Larochette, Wawrziczny, Papo, Pasquier y Antoine, 2019).

En el caso particular de la soledad, estos sentimientos emergen debido a que las responsabilidades de la persona reducen su tiempo libre y disminuyen el contacto físico y emocional con el ser amado (Leszko, Iwański y Bugajska, 2020). Aunado a lo anterior, la pareja del paciente llega a sentirse desprotegida y vulnerable, puesto que siempre es ella la que debe hacerse cargo de las dificultades. Además, aparece también la soledad como un efecto de perder relaciones importantes en la red social de la pareja, de los cambios en la estructura del grupo social y del nivel de la relación y el apoyo percibido, que llegan a considerarse como escasos o insignificantes (Cruz, Sánchez y Castro, 2020).

De acuerdo con Peplau y Perlman (1982), la soledad se caracteriza por ser el resultado de un número limitado de relaciones interpersonales estrechas; una experiencia subjetiva que se logra evidenciar por el aislamiento social de la persona 
y sus bajos niveles de contacto social, así como una sensación estresante y displacentera para quien la experimenta.

Justamente, la soledad constituye un sentimiento negativo que se ha asociado a variables tales como depresión, ansiedad, insatisfacción, infelicidad, estrés (Domenech, Mundò, Haro y Rubio, 2019; Xia y Li, 2018), enfermedades cardiovasculares, trastornos de la alimentación, problemas de sueño (Mann et al., 2017) y muchas más experiencias psicológicas perjudiciales que pueden significar amenaza o pérdida, haciendo que las personas tiendan a evaluar las causas, significados y consecuencias de esas experiencias, o bien a recordar constantemente los errores y equivocaciones pasadas que no se han resuelto satisfactoriamente (Nolen-Hoeksema, Vine y Gilbert, 2013). Martin y Tesser (1996) denominaron a estos procesos de reflexión y evaluación constante como rumiación; mientras que autores como Bernstein, Heeren y McNally (2017) la definen como un estilo cognitivo caracterizado por pensamientos repetitivos, recurrentes, intrusivos e incontrolables que interrumpen el diario vivir de la persona. Otros autores la consideran como un conjunto de pensamientos y comportamientos que centran la atención de la persona en estados de ánimo negativos, en sus causas y consecuencias y en las autoevaluaciones relacionadas con ellos (Eisma y Stroebe, 2017; Smith, Mason y Lavender, 2018). A partir de lo anterior, se presume que la soledad influye en la medida en la que la atención se centra en los pensamientos negativos resultantes de provocaciones y estresores pasados (Zawadzki, Graham y Gerin, 2013), lo que quita a la persona el deseo de entablar relaciones con otros; no obstante, las investigaciones al respecto han sido escasas y la población foco analizada no ha sido la de cuidadores de pacientes con ciertas enfermedades.

Por último, en algunos estudios se ha encontrado una asociación entre la rumiación y el estrés (Du, Huang, An y Xu, 2018; Malin y Littlejohn, 2015; Zawadzki et al., 2013), señalándose que los estados cognitivos repetitivos y persistentes aumentan la activación fisiológica del sistema nervioso simpático, generando así un estado constante de perturbación y alerta al no haber una forma eficaz de lidiar con los conflictos pasados que no

Instituto de Investigaciones Psicológicas - Universidad Veracruzana ISSN impreso: 1405-1109 han sido del todo superados. De esta manera se puede entender la relación entre el estrés y la rumiación como una conexión bilateral, puesto que los pensamientos repetitivos e intrusivos pueden generar estrés, pero a su vez altos niveles de este pueden desarrollar pensamientos constantes e incontrolables en la persona. Es así como dicho estado constante de alerta incrementa la tensión y los niveles de glucosa, dilata los bronquios y acelera los latidos del corazón, acrecentando el riesgo de producir daños a la salud.

Según Martínez, Grau y Rodríguez (2017), las labores que debe cumplir la pareja de la persona enferma, sumadas a la falta de control de la situación, traen como resultado una sobrecarga y sentimientos y actitudes negativas hacia esta última, así como desmotivación, cansancio excesivo e irritabilidad, lo que finalmente se traduce en niveles elevados de estrés.

Cabe señalar que hay dos clases de estrés: el crónico y el agudo. El primero es el resultado de la respuesta de alerta y de preocupación constante y permanente; en cambio, el segundo está más relacionado a una angustia de corta duración y limitada temporalmente (Banegas y Sierra, 2017). Tanto el estrés y la rumiación acarrean consecuencias negativas para la salud física y emocional de la persona, disminuyendo su bienestar subjetivo y la satisfacción que tiene con la vida (Denovan y Macaskill, 2016; Flórez y Sánchez, 2020; Gillett y Crisp, 2017; Sariçam, 2016), bajo la lógica de que las demandas que trae el cuidado del paciente son estresores potenciales que desestabilizan su bienestar, dado que la persona asume una tarea no retribuida y en ocasiones no esperada (Bethancourt, Bethancourt, Moreno y Suárez, 2014). En el caso de la rumiación, la persona ve alterada su vida al concentrar sus energías en los aspectos negativos y las consecuencias de la enfermedad, perturbándola y comprometiendo sus emociones, todo ello aunado a la fuerte carga física, psicológica y social. Asimismo, la desesperanza y tensión son sentimientos recurrentes al verse incapacitada para pronosticar cuándo ella y su pareja volverán a sentirse mejor (Bethancourt et al., 2014).

Por otra parte, como se ha reportado en estudios previos, la soledad es un importante factor de riesgo de emociones y estados cognitivos nega- 
tivos, incluidas la depresión y la ansiedad, que reducen aún más el bienestar de la pareja (Gan, Xie, Duan, Deng y Yu, 2015). Sumado a ello, los cuidadores, al no desarrollar y afianzar sus relaciones interpersonales importantes, experimentan menos felicidad ya que, tal como lo señala Domínguez (2016), el tiempo de esparcimiento, las actividades recreativas, la calidad de las relaciones familiares y la comunicación constante se ven disminuidos, afectando así a la persona y a su red social de apoyo, por lo que aquella se ve cada vez más aislada y abrumada.

Es así como se puede apreciar el rol del cuidador primario (pareja) y el riesgo que genera para su salud atender las necesidades del miembro enfermo, y más aún en México, donde las personas que cumplen estas funciones llevan a cabo frecuentemente otras actividades inherentes a su vida cotidiana, como por ejemplo el cuidado de los hijos y las labores del hogar o del trabajo, entre otras (Secinti et al., 2019). Pese a todo lo dicho, las investigaciones a este respecto no han sido completas, además de que la atención médica y psicológica de esta problemática también son limitadas.

Otra variable que interviene en la dinámica del cuidador es el sexo, pues se ha visto que existen diferencias importantes en la manera en la que hombres y mujeres prestan ayuda al paciente enfermo. En algunos estudios se ha reportado que son las mujeres las que asumen en mayor medida el rol de cuidadoras, en comparación con los hombres (Jiménez y Moya, 2018). Asimismo, se tiene asociada la imagen de la mujer con la posesión de características útiles para el cuidado, como entrega, expresión de afecto, abnegación y generosidad. Por su parte, los hombres tienden en menor medida a llevar a cabo tareas diarias que se consideran sumamente demandantes, como limpiar, cocinar o atender a la persona enferma, por lo que, a medida que avanza y se intensifica la enfermedad y las necesidades de cuidado, suelen abandonar su rol de cuidadores (Sharma, Chakrabart y Grover, 2016).

A partir de lo mencionado, los objetivos del presente estudio fueron: 1) explorar las diferencias entre hombres y mujeres, parejas de pacientes con cardiopatía, respecto a la soledad, la rumiación, el estrés y el bienestar subjetivo experimentados; 2) identificar la relación entre la soledad y la

Instituto de Investigaciones Psicológicas - Universidad Veracruzana rumiación y el estrés, y 3) identificar el nivel de predicción de la soledad, la rumiación y el estrés sobre el bienestar subjetivo, todo ello en personas con una pareja con algún tipo de cardiopatía.

\section{MÉTODO}

\section{Participantes}

Se empleó una muestra no probabilística por cuota (Hernández, Fernández y Baptista, 2010) de 130 participantes (65 hombres y 65 mujeres), residentes de la Ciudad de México, cuyas edades oscilaban entre 19 a 68 años $(\mathrm{M}=44.02$, D.E. $=11.43)$. En cuanto a su escolaridad, 36 participantes tenían estudios de secundaria, 46 de preparatoria y 47 de licenciatura; una persona no respondió. Con respecto al tiempo de vivir con su pareja, este varió de 10 meses a 50 años; en cuanto al estado civil reportado, $28.5 \%$ vivían en unión libre y $71.5 \%$ estaban casados.

\section{Instrumentos}

Escala de Soledad (DiTomasso y Spinner, 1992). Adaptada por Granillo y Sánchez (en proceso de evaluación), evalúa la experiencia subjetiva de displacer, estrés y dolor causados por las deficiencias percibidas en las interacciones socioafectivas o a la falta de intimidad interpersonal (Peplau y Perlman, 1982). Consta de 31 reactivos distribuidos en cinco factores, cuyas respuestas se evalúan en una escala tipo Likert de cinco puntos que indican el grado de acuerdo y que va de 1 ("Totalmente en desacuerdo") a 5 ("Totalmente de acuerdo"). Sus factores se denominan Acompañamiento de la pareja, Acompañamiento de los amigos, Acompañamiento de la familia, Sentimientos de vacío y Añoranza. Tales factores explican $52.05 \%$ de la varianza total, y sus coeficientes alfa de Cronbach $(\alpha)$ van de moderados a moderados altos, siendo el total de .74.

\section{Escala de Rumiación}

(Flórez y Sánchez, en proceso de evaluación).

Este instrumento está constituido por diez ítems en formato tipo Likert de cinco puntos que indican 
el grado de acuerdo con las afirmaciones, donde 1 indica "Totalmente en desacuerdo" y 5 "Totalmente de acuerdo". Los ítems se agrupan en dos factores que explican $67.29 \%$ de la varianza, con un $\alpha$ total de .91. El primer factor se denomina Malestar (cinco reactivos, $\alpha$ de .89), y el segundo Reflexión obsesiva (cinco reactivos y $\alpha$ de .84).

Escala de Estrés (Flórez y Sánchez, 2019).

Consta de trece ítems con formato tipo Likert de cinco puntos que indican el grado de acuerdo con las afirmaciones, donde 1 indica "Totalmente en desacuerdo" y 5 "Totalmente de acuerdo"; con $\alpha$ total de.82; dichos ítems están distribuidos en dos factores que explican $50.96 \%$ de la varianza: Descontrol (seis reactivos y $\alpha$ de .77) y Control (siete reactivos y $\alpha$ de .72).

Escala de Bienestar Subjetivo (Diener et al., 2009). Adaptada y validada en México por Granillo, Zepeda y Sánchez (en proceso de evaluación), está compuesta por tres subescalas con formato de respuesta tipo Likert con opciones de respuesta que indican grados de frecuencia. La primera, la subescala de Experiencia positiva y negativa, está compuesta por once reactivos que se distribuyen en dos factores que explican $58.987 \%$ de la varianza: Experiencia positiva ( $\alpha$ de .85 ) y Experiencia negativa ( $\alpha$ de .81 ); la segunda, Prosperidad, consta de ocho reactivos que explican $58.12 \%$ de la varianza y con un $\alpha$ total de .89 , y Satisfacción con la vida, está integrada por cinco reactivos que explican $63.78 \%$ de la varianza y con $\alpha$ total de .84 .

\section{Procedimiento}

Los cuestionarios fueron aplicados por estudiantes de Psicología, quienes acudieron a diversos lugares (hospitales, clínicas, oficinas o escuelas) en los que se podían encontrar personas que hubieran tenido o tuvieran actualmente una relación de pareja con alguien que padeciera alguna cardiopatía, para que de manera voluntaria, anónima y confidencial contestaran los referidos instrumentos. Se les dio instrucciones para su llenado, se resolvieron sus dudas y se pusieron a su disposición los resultados.

\section{Análisis de datos}

Para lograr responder los objetivos planteados en un primer momento, se realizó una prueba de normalidad de Kolmogorov-Smirnov con el fin de conocer la distribución de las variables en la muestra bajo estudio y escoger el tipo de análisis estadístico a utilizar. Después se llevó a cabo una prueba U de Mann-Whitney para cumplir el primer objetivo, una correlación de Spearman para el segundo y una regresión lineal para el tercero, todo ello mediante el programa estadístico SPSS, versión 20.

\section{RESULTADOS}

La prueba de normalidad Kolmogorov Smirnov mostró que no había normalidad en los datos $(p<.001)$, por lo que se decidió realizar una prueba U de Mann Whitney para cubrir el primer objetivo. Como se aprecia en la Tabla 1, los resultados de este primer análisis mostraron que no había diferencias estadísticamente significativas entre hombres y mujeres $(p>.05)$, parejas de pacientes con cardiopatía. Por consiguiente, los siguientes análisis se hicieron con toda la muestra y no por hombres y mujeres.

Así, para dar solución al segundo objetivo, que fue identificar la relación entre la soledad con la rumiación y el estrés, en la Tabla 2 se muestra que cuando las parejas de pacientes con cardiopatía se sienten más acompañadas por su pareja y amistades, más control experimentan ante las situaciones de estrés, así como menos malestar (sufrimiento por pensar en eventos negativos) y pensamientos obsesivos sobre los errores que creen que han cometido en el pasado; cuando se sienten más acompañadas por parte de su familia, más control ante el estrés experimentan, y a más sentimientos de vacío por no tener personas con quien compartir momentos importantes, más reflexión obsesiva sobre problemas pasados, más malestar, más descontrol y menos control ante el estrés experimentan. Por último, en la medida en que estas personas más desean entablar relaciones personales duraderas - pues carecen de ellas-, más reflexión obsesiva y malestar padecen. 
Tabla 1. Diferencias en soledad, rumiación, estrés y bienestar subjetivo por sexo.

\begin{tabular}{|c|c|c|c|c|c|c|c|c|c|c|c|c|c|}
\hline & \multicolumn{5}{|c|}{ Soledad } & \multicolumn{2}{|c|}{ Rumiación } & \multicolumn{2}{|c|}{ Estrés } & \multicolumn{4}{|c|}{ Bienestar subjetivo } \\
\hline & 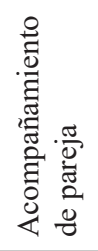 & 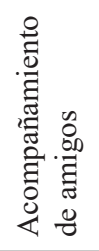 & 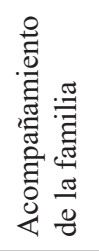 & 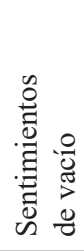 & 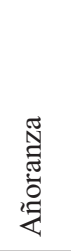 & $\begin{array}{l}\frac{\vec{w}}{w_{0}} \\
\frac{0}{\pi} \\
\sum\end{array}$ & 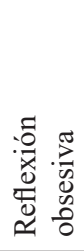 & $\begin{array}{l}\overline{\text { on }} \\
\text { 音 }\end{array}$ & 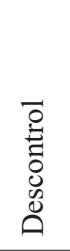 & 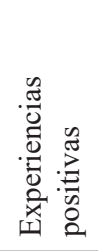 & 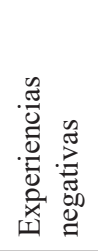 & 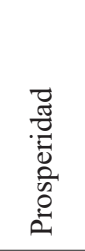 & 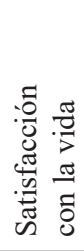 \\
\hline Mujeres & 66 & 58 & 63 & 67 & 62 & 60 & 60 & 57 & 71 & 62 & 71 & 62 & 66 \\
\hline Hombres & 61 & 66 & 60 & 60 & 65 & 59 & 69 & 72 & 69 & 67 & 67 & 68 & 64 \\
\hline Z & -1.9 & -1.4 & -66 & -1.8 & -91 & -33 & -1.8 & -1.7 & -85 & -1.2 & -38 & -1.0 & -43 \\
\hline$p$ & .05 & .15 & .50 & .06 & .35 & .73 & .05 & .07 & .39 & .21 & .69 & .29 & .66 \\
\hline
\end{tabular}

Tabla 2. Correlación entre soledad con rumiación y estrés.

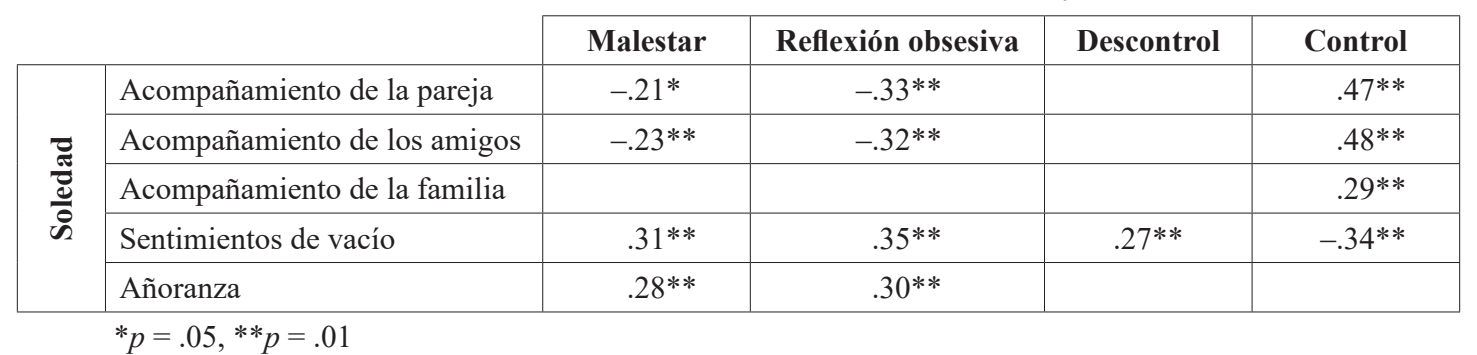

En lo que respecta al tercer objetivo, esto es, identificar el nivel de predicción de la soledad, la rumiación y el estrés con el bienestar subjetivo, en la Tabla 3 se muestra que las parejas de pacientes con cardiopatía, en la medida que se sienten parte de un círculo social cercano conformado por su pareja, se sienten más positivas y satisfechas con su vida, y cuando perciben el acompañamiento de su familia se sienten más prósperas y experimentan más emociones positivas. Por el contrario, cuando los participantes vivencian sentimientos de vacío, menos prósperos se sienten, y cuando tienen un deseo incumplido de adquirir relaciones interpersonales cercanas y duraderas, más experiencias negativas aprecian, lo que trae a su vez menos prosperidad y satisfacción en sus vidas; por el contrario, cuando se sienten más capaces de controlar las situaciones estresantes del día a día, más prosperidad, más satisfacción con la vida y más emociones positivas experimentan, disminuyendo así las emociones negativas, como se puede observar en la Tabla 3.

Tabla 3. Análisis de regresión para las variables criterio para predecir el bienestar subjetivo.

\begin{tabular}{|c|c|c|c|c|c|}
\hline \multicolumn{2}{|r|}{ Variables predictoras } & $\beta$ & $p$ & $\mathbf{R}^{2}$ & Bienestar subjetivo \\
\hline \multirow{9}{*}{ 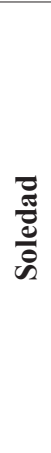 } & \multirow{2}{*}{ Acompañamiento pareja } & .24 & .001 & .47 & Experiencia positiva \\
\hline & & .31 & .000 & .55 & Satisfacción con la vida \\
\hline & \multirow{3}{*}{ Acompañamiento familia } & .28 & .045 & .47 & Experiencia positiva \\
\hline & & .28 & .037 & .49 & Prosperidad \\
\hline & & .38 & .006 & .55 & Satisfacción con la vida \\
\hline & Sentimiento de vacío & .17 & .025 & .52 & Experiencia negativa \\
\hline & \multirow{3}{*}{ Añoranza } & -.17 & .010 & .47 & Experiencia positiva \\
\hline & & -.23 & .000 & .49 & Prosperidad \\
\hline & & -.18 & .004 & .55 & Satisfacción con la vida \\
\hline \multirow{4}{*}{ 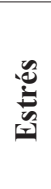 } & \multirow{4}{*}{ Control } & .29 & .010 & .47 & Experiencia positiva \\
\hline & & -.22 & .034 & .52 & Experiencia negativa \\
\hline & & .40 & .000 & .49 & Prosperidad \\
\hline & & .41 & .000 & .55 & Satisfacción con la vida \\
\hline
\end{tabular}




\section{DISCUSIÓN}

Respecto al primer objetivo, no se encontraron diferencias estadísticamente significativas entre los hombres y las mujeres, parejas de pacientes con cardiopatías, lo que puede explicarse a partir de la interdependencia que ha construido la pareja, y que permite a ambos miembros sincronizar sus emociones, pensamientos y conductas, principalmente cuando hay en uno de ellos una enfermedad que exige que sean un equipo (Osorio, Chávez, Silva, Parrello y Iorio, 2017; Sánchez, 2016), especialmente cuando se habla de las cardiopatías. Tal como lo expresan Torres, Carreño y Chaparro (2017), las cardiopatías son afecciones que alteran profundamente el entorno inmediato del paciente, afectando de igual manera a las personas que se hacen cargo de este y que asumen la responsabilidad y la labor de cuidado, sin importar su sexo, puesto que tanto hombres y mujeres sienten la necesidad de contribuir al mejoramiento y recuperación de su pareja. Es así como dichos hallazgos permiten entender por qué no se hallaron diferencias según el sexo, lo que trajo por consecuencia analizar las variables tomando en cuenta al total de la muestra, y no a hombres y mujeres por separado.

En cuanto al segundo objetivo, los datos muestran que los participantes que gozaban de relaciones interpersonales estables y enriquecedoras (pareja y amigos) experimentaron más control y menos rumiación, debido posiblemente a que las relaciones íntimas tienen un gran valor en la vida del ser humano (Thornton y Freedman, 1982), toda vez que contribuyen generando emociones positivas, como la felicidad, el optimismo, la tolerancia, el sentido del humor y la creatividad, entre otras (Flórez y Sánchez, 2019), las cuales generan estrategias que hacen posible sopesar las molestias que trae consigo la enfermedad (Varela y Barrón, 2016). A su vez, en la mayoría de los casos, la pareja cumple la función de protección y resguardo ante las adversidades lo que dificulta evocar pensamientos negativos.

De la misma manera, se observa que las personas que pertenecen a una familia se encuentran capacitadas para controlar situaciones de estrés -aunque en menor medida que cuando se sienten en compañía de su pareja o de amigos-. Debe se-

Instituto de Investigaciones Psicológicas - Universidad Veracruzana ISSN impreso: 1405-1109 ñalarse que este vínculo funciona como un sostén para sobreponerse a las demandas que se derivan de cuidar a la pareja enferma; no obstante, es claro que la pareja y los amigos ejercen mayor influencia. De hecho, la forma en la que estos y la familia controlan el estrés radica en que ellos son una importante fuente de apoyo, lo que disminuye la sobrecarga de trabajo y los sentimientos y pensamientos negativos y hostiles (Zawadzki et al., 2013), así como la posible sensación de desprotección (Feldberg et al., 2011).

Por otra parte, cuando los participantes sienten que no tienen personas cercanas con quienes compartir los momentos importantes de su vida, experimentan más malestar y mayores niveles de reflexión obsesiva y descontrol ante el estrés. De igual manera, suelen desear persistentemente entablar relaciones duraderas, lo que trae como consecuencia una mayor rumiación debido a que enfrentar la enfermedad de su pareja y todas las responsabilidades que trae consigo produce una sensación de saturación física y psicológica. Situación que aumenta los niveles de estrés y deteriora la calidad de vida de ambos miembros (Feldberg et al., 2011). Debe señalarse que los participantes, al verse expuestos recurrentemente al estresor, buscan una salida a través de sus pensamientos, al tiempo que desean compartir momentos agradables y volver a lo que eran antes junto a su pareja, amigos y familia.

En el tercer objetivo se logró evidenciar el nivel de predicción de la soledad y el estrés en el bienestar subjetivo, lo que muestra que los participantes experimenten más satisfacción con sus vidas, más prosperidad y positividad al contar con la compañía de personas cercanas, como su pareja y familia, puesto que, tal como se señaló antes, las relaciones interpersonales contribuyen al bienestar del ser humano al generar una diferencia sustancial respecto a la percepción que el sujeto tiene de sí mismo y de su sentido de vida y propósito (Lacunza y Contini, 2016), ya que favorecen el bienestar y la felicidad de su pareja y de sus seres queridos. Por el contrario, los sentimientos de vacío predicen una menor prosperidad, menos necesidad de hallar un vínculo afectivo significativo y baja satisfacción con la vida, así como más emociones negativas. Tales hallazgos se logran explicar primeramente con base en el valor que tienen 
las relaciones interpersonales inmediatas, pues son estas las que más importancia tienen para el ser humano en tanto que le brindan apoyo y compañía en los momentos más difíciles; al no tenerlas, aumentan las emociones negativas, como la tristeza y el miedo. En segundo lugar, encontrarse continuamente buscando vínculos y relaciones de apoyo debilita emocionalmente al sujeto, más aún cuando debe enfrentarse al cuidado de un enfermo (Schulz et al., 2018). Aunado a lo anterior, también se logra experimentar un incremento en las emociones negativas en razón de que estar cerca de una persona que padece una enfermedad crónica -y más aún cuando es la propia pareja-produce sentimientos de decaimiento, tristeza, frustración e incertidumbre (Mendes, Fortes, Ribeiro, Astres y Barros, 2019). En efecto, ya Palacios y Jiménez (2008) han apuntado que los cuidadores pasan por las mismas etapas de duelo que el paciente: negación, cólera, negociación, depresión y aceptación, lo que está estrechamente relacionado con la disminución de la satisfacción y la felicidad.

Así entonces, cuando la pareja del paciente cardiópata siente que no tiene a alguien con quien compartir su vida para expresar sus sentimientos y pasar un tiempo de calidad, es posible que, como cuidadora, resulte ser la más afectada en la esfera emocional y social, lo que, según los niveles de sobrecarga de trabajo, la lleva a desatender su propia salud. El riesgo de colapsar en su bienestar subjetivo es inminente cuando proporciona acompañamiento activo a su pareja con acciones como llevar el control de la toma de medicamentos; los cambios y la adopción de hábitos alimenticios; el fomento de la actividad física; las visitas rutinarias a las consultas médicas, y la motivación y el apoyo emocional al paciente para contribuir al mejoramiento de su calidad de vida al no disponer de una red de apoyo importante (Bethancourt et al., 2014). Aunado a lo anterior, el cuidador se ve afectado física, psicológica y cognitivamente a causa de la alteración de su rol habitual, la disminución en sus interacciones sociales y la falta de respaldo para el apoyo y el cuidado que requiere el paciente (Xie et al., 2016).
Finalmente, quienes logran controlar el estrés, más prosperidad, satisfacción y positividad sienten tener en sus vidas, dado que, tal como lo expresan Verdugo et al. (2012), son las personas con mejores habilidades para el afrontamiento y el dominio de su entorno; además, al ejecutar un adecuado control de la tensión se sienten más satisfechos y gozan de una adecuada salud mental, así como también de la ausencia de tensiones, depresión, afectos negativos y ansiedad.

Es así como, la presente investigación logró cumplir con los objetivos establecidos al evidenciar el efecto que tienen la enfermedad, la soledad, la rumiación y el estrés en el bienestar subjetivo de ambos miembros de la pareja, y sobre todo del cuidador, afectando su vivencia anímica y trayendo consigo soledad, rumiación y estrés que posteriormente alterarán su experiencia de bienestar subjetivo, ya que los diferentes tipos de cardiopatías suele ser incapacitantes, de larga duración y difíciles de sobrellevar para ambas partes. El cuidador debe hacerse a la idea de que asumirá el cuidado de su pareja de manera permanente, lo que tendrá un considerable efecto en su estilo de vida.

Este trabajo cobra relevancia al ser México uno de los países más afectados por esta enfermedad y por el olvido en que se tiene al cuidador primario, el muchas veces llamado "paciente silencioso".

Para futuras investigaciones en esta línea se sugiere estudiar la soledad junto con otras variables evidentes en la dinámica relacional; por ejemplo, la comunicación, la satisfacción marital o el manejo del poder, entre muchas otras, para así, evidenciar cómo se comporta dicha variable en la interacción continua con la pareja. Igualmente, se propone ahondar en el cuidador como actor principal a fin de evidenciar las afectaciones a las cuales está expuesto.

Cabe mencionar que una de las limitaciones del estudio fue que se asumió a la pareja como el cuidador primario, aunque dentro del núcleo familiar puede ser cualquier otra persona la que asuma esta responsabilidad y se haga cargo de la persona enferma. 


\section{AGRADECIMIENTOS}

Investigación realizada gracias al Programa UNAM-PAPIIT IN304919 del proyecto: "Factores protectores y de riesgo a la salud en parejas sanas y con enfermedad crónico-degenerativa".

Citación: Flórez-Rodríguez, Y.N. y Sánchez-Aragón, R. (2022). Soledad, rumiación y estrés como precursores del bienestar subjetivo en las parejas de pacientes con cardiopatía. Psicología y Salud, 32(1), 81-91. https://doi.org/10.25009/pys.v32i1.2713.

\section{REFERENCIAS}

Banegas, A. y Sierra, L. (2017). Variables bioquímicas e inmunológicas en pacientes con estrés agudo o crónico. Medisan, 21(8), 1018-1026.

Bernstein, E., Heeren, A. y McNally, R. (2017). Unpacking rumination and executive control: a network perspective. Clinical Psychological Science, 5(5), 816-826.

Bethancourt, Y., Bethancourt, J., Moreno, Y. y Suárez, A. (2014). Evaluación del bienestar psicológico en cuidadores primarios de pacientes oncológicos en la fase de cuidados paliativos. Mediciego, 20(2), 1-7.

Cruz D., J., Sánchez, R. y Castro, V. (2020). Soledad y salud: ¿cómo se relacionan y difieren según la edad y la escolaridad? Psicología y Salud, 30(2), 231-241.

Denovan, A. y Macaskill, A. (2016). Stress and subjective well-being among first year UK undergraduate students. Journal of Happiness Studies, 18(1), 505-525.

Diener, E., Wirtz, D., Tov, W., Kim, C., Choi, D., Oishi, S. y Biswas, R. (2009). New measures of well-being: Flourishing and positive and negative feelings. Social Indicators Research, 39(1), 247-266. Doi: 10.1007/s11205-009-9493-y.

DiTomasso, E. y Spinner, B. (1993). The development and initial validation of the Social and Emotional Loneliness Scale for Adults (SELSA). Personality and Individual Differences, 14(1), 127-134.

Domenech, J., Mundò, J., Haro, J. y Rubio, M. (2019). Anxiety, depression, loneliness and social network in the elderly: longitudinal associations from The Irish Longitudinal Study on Ageing (TILDA). Journal of Affective Disorders, $246(1)$, 82-88.

Domínguez, M. (2016). Bienestar en cuidadores familiares de adultos mayores. Revista Iberoamericana de Diagnóstico y Evaluación, 41(1), 104-117.

Du, J., Huang, J., An, Y. y Xu, W. (2018). The relationship between stress and negative emotion: The mediating role of rumination. Clinical Research and Trials, 4(1), 1-5. Doi: 10.15761/CRT.1000208.

Eisma, M. y Stroebe, M. (2017). Rumination following bereavement: an overview. Bereavement Care, 36(2), 58-64. Doi: 10.1080/02682621.2017.1349291.

Feldberg, C., Tartaglini, M., Clemente, M., Petracca, G., Cáceres, F. y Stefani, D. (2011). Vulnerabilidad psicosocial del cuidador familiar. Creencias acerca del estado de salud del paciente neurológico y el sentimiento de sobrecarga. Neurología Argentina, 3(1), 11-16. Doi: 10.1016/S1853-0028(11)70003-9.

Flórez Y., N. y Sánchez, R. (En proceso de evaluación). Midiendo el estrés y la rumia: su percepción de reto vs. amenaza. Avances en Psicología Latinoamericana.

Flórez Y., N. y Sánchez, R. (2019). Optimismo, resiliencia, sentido del humor y la salud de personas con pareja. Pensando Psicología, 15(26), 1-24.

Flórez Y., N. y Sánchez, R. (2020). El estrés visto como reto o amenaza y la rumia. Factores de riesgo a la salud. Salud y Administración, 7(20), 17-27.

Gan, P., Xie, Y., Duan, D., Deng, Q. y Yu, X. (2015). Rumination and loneliness independently predict six-month later depression symptoms among Chinese elderly in nursing homes. PLoS One, 10(9), 1-10. Doi: 10.1371/journal.pone.0137176.

Gillett, J. y Crisp, D. (2017). Examining coping style and the relationship between stress and subjective well-being in Australia's "sandwich generation". Australasian Journal on Ageing, 36(3), 222-227. Doi: 10.1111/ajag.12439.

Granillo L., F. y Sánchez, R. (En proceso de evaluación). Medición de la soledad en México y su relación con el apego: Ciencia UAT.

Granillo, A.D., Zepeda, G. y Sánchez, R. (En proceso de evaluación). Bienestar subjetivo: Medición integral y validación en México: DUAZARY.

Hernández, R., Fernández, C. y Baptista, P. (2010). Metodología de la investigación. Lima: McGraw-Hill.

Instituto de Investigaciones Psicológicas - Universidad Veracruzana

ISSN impreso: 1405-1109
Psicología y Salud, Vol. 32, Núm. 1: 81-91, enero-junio de 2022

https://doi.org/10.25009/pys.v32i1.2713 
Instituto Nacional de Estadística y Geografía (2017). Características de las defunciones registradas en México durante 2017. Ciudad de México: INEGI. Recuperado de https://www.inegi.org.mx/contenidos/saladeprensa/boletines/2018/EstSociodemo/DEFUNCIONES2017.pdf.

Jackson, J., Leslie, C. y Hondorp, S. (2018). Depressive and anxiety symptoms in adult congenital heart disease: 2 Prevalence, health impact and treatment. Progress in Cardiovascular Diseases, 61(3-4), 294-299. Doi: 10.1016/j.pcad.2018.07.015.

Jiménez, I. y Moya, M. (2018). La cuidadora familiar: sentimiento de obligación naturalizado de la mujer a la hora de cuidar. Enfermería Global, 17(1), 420-447.

Lacunza, A. y Contini, E. (2016). Relaciones interpersonales positivas: los adolescentes como protagonistas. Psicodebate, 16(2), 73-94. Doi: 10.18682/pd.v16i2.598.

Larochette, C., Wawrziczny, E., Papo, D., Pasquier, F. y Antoine, P. (2019). An acceptance, role transition, and couple dynamics-based program for caregivers: A qualitative study of the experience of spouses of persons with young-onset dementia. Dementia, 18(6), 1-18.

Leszko, M., Iwański, R. y Bugajska, B. (2020). Prisoners of care: The experience of loneliness among caregivers of individuals with Alzheimer's disease. Paedagogia Christiana, 45(1), 171-184.

Malin, K. y Littlejohn, G. (2015). Rumination modulates stress and other psychological processes in fibromyalgia. European Journal of Rheumatology, 2(4), 143-148. Doi: 10.5152/eurjrheum.2015.0005.

Mann, F., Bone, J., Lloyd-Evans, B., Frerichs, J., Pinfold V., M.R., Wang, J. y Johnson, S. (2017). A life less lonely: the state of the art in interventions to reduce loneliness in people with mental health problems. Social Psychiatry and Psychiatric Epidemiology, 52(1), 627-638.

Martin, L. y Tesser, A. (1996). Some ruminative thoughts. En R. S. Wyer, Jr. (Ed.): Ruminative thoughts (pp. 1-47). Mahwah, NJ: Lawrence Erlbaum.

Martínez, J., Fuertes, A., Orgaz, B., Vicario, I. y González, E. (2014). Vínculos afectivos en la infancia y calidad en las relaciones de pareja de jóvenes adultos: el efecto mediador del apego actual. Anales de Psicología, 30(1), 211-220.

Martínez, L., Grau, Y. y Rodríguez, R. (2017). Estrategias de afrontamiento al estrés en cuidadores de pacientes en hemodiálisis. Enfermería Nefrológica, 20(2), 139-148.

Mendes, P., Fortes, M., Ribeiro, A., Astres, M. y Barros, R. (2019). Physical, emotional and social burden of elderly patients' informal caregivers. Acta Paulista de Enfermagem, 32(1), 87-94.

Nolen-Hoeksema, S., Vine, V. y Gilbert, K. (2013). Rumination and emotions. En C. Mohiyeddini, S. Bauer y M. Eysenck (Eds.): Handbook of psychology of emotions: Recent theoretical perspectives and novel empirical findings (pp. 120-149). Hauppauge, NY: Nova Science Pub Inc.

Organización Mundial de la Salud (2017). Enfermedades cardiovasculares. Ginebra: OMS. Recuperado de https://www.who.int/ es/news-room/fact-sheets/detail/cardiovascular-diseases-(cvds).

Osorio, M., Chávez, S., Silva, G., Parrello, S. e Iorio, I. (2017). Parejas de pacientes con una enfermedad crónica. Estudio narrativo sobre la hemofilia. Revista Electrónica de Psicología Iztacala, 20(2), 548-569.

Palacios, X. y Jiménez, K. (2008). Estrés y depresión en cuidadores informales de pacientes con trastorno afectivo bipolar. Avances en Psicología Latinoamericana, 26(2), 195-210.

Peplau, L.A. y Perlman, D. (1982). Perspectives on loneliness. En L. A. Peplau y D. Perlman (Eds.): Loneliness: A sourcerbook of current theory, research and therapy (pp. 1-18). New York: Wiley.

Sánchez, R. (2016). La pareja romántica en México. Ciudad de México: Universidad Nacional Autónoma de México.

Sariçam, H. (2016). Examining the relationship between selfrumination and happiness: The mediating and moderating role of subjective vitality. Universitas Psychologica, 15(2), 383-396. Doi: 10.11144/Javeriana.upsy15-2.errh.

Schulz, R., Beach, S., Friedman, E., Martsolf, G., Rodakowski, J. y James, E. (2018). Changing structures and processes to support family caregivers of seriously ill patients. Journal of Palliative Medicine, 21(2), 36-42. Doi:10.1089/jpm.2017.0437.

Secinti, E., Rand, K., Johns, S., O’Neil, B., Helft, P., Shahda, S. y Mosher, C. (2019). Social correlates of mental health in gastrointestinal cancer patients and their family caregivers: Exploring the role of loneliness. Supportive Care in Cancer, 27(1), 2077-2086.

Sharma, N., Chakrabart, S. y Grover, S. (2016). Gender differences in caregiving among family -caregivers of people with mental illnesses. World Journal of Psychiatry, 6(1), 7-17. Doi: 10.5498/wjp.v6.i1.7.

Smith, K., Mason, T. y Lavender, J. (2018). Rumination and eating disorder psychopathology: A meta-analysis. Clinical Psychology Review, 61(3), 9-23.

Thornton, A. y Freedman, D. (1982). Changing attitudes toward married and single life. Family Planning Perspectives, 14(6), 297-303. Doi: $10.2307 / 2134600$.

Torres, X., Carreño, S. y Chaparro, L. (2017). Factores que influencian la habilidad y sobrecarga del cuidador familiar del enfermo crónico. Revista de la Universidad Industrial de Santander, 49(2), 330-338. 
Valentín, A. (2018). Cardiopatías congénitas en edad pediátrica, aspectos clínicos y epidemiológicos. Revista Médica Electrónica, 40(4), 1083-1099. http://www.revmedicaelectronica.sld.cu/index.php/rme/article/view/2479/3971.

Varela, I. y Barrón, A. (2016). La influencia de la pareja en el ajuste a la enfermedad cardiaca. Acta de Investigación Psicológica, $6(2), 2459-2468$.

Verdugo, J., Ponce, B., Guardado, R., Meda, R.M., Uribe, J. y Guzmán, J. (2013). Estilos de afrontamiento al estrés y bienestar subjetivo en adolescentes y jóvenes. Revista Latinoamericana de Ciencias Sociales, Niñez y Juventud, 11(1), 79-91.

Xia, N. y Xi, H. (2018). Loneliness, social isolation, and cardiovascular health. Antioxidants \& Redox Signaling, $28(9)$, 837-851. Doi: 10.1089/ars.2017.7312.

Xie, H., Cheng, C., Tao, Y., Zhang, J., Robert, D., Jia, J. y Su, Y. (2016). Quality of life in Chinese family caregivers for elderly people with chronic diseases. Health and Quality of Life Outcomes, 99(14), 1-9. Doi: 10.1186/s12955-016-0504-9.

Zavala, L., Verdejo, M. y Díaz, R. (2015). Relación entre estrés y estilos de afrontamiento con el desarrollo de la cardiopatía isquémica. Psicología Iberoamericana, 23(1), 7-15.

Zawadzki, M., Graham, J. y Gerin, W. (2013). Rumination and anxiety mediate the effect of loneliness on depressed mood and sleep quality in college students. Health Psychology, 32(2), 212-222. doi: 10.1037/a0029007. 